\title{
Pressure Point Threshold and ME/CFS comorbidity as Indicators of Physiotherapy Response in Fibromyalgia
}

\author{
Francisco Javier Falaguera-Vera ${ }^{1}$, María Garcia-Escudero ${ }^{2,3}$, Javier Bonastre-Férez ${ }^{4}$, Mario \\ Zacarés ${ }^{5}$ and Elisa Oltra ${ }^{6,7 *}$ \\ 1 Escuela de Doctorado, Universidad Católica de Valencia San Vicente Mártir, 46008 Valencia, Spain; \\ fj.falaguera@ucv.es \\ 2 School of Health Sciences, Universidad Católica de Valencia San Vicente Mártir, 46900 Valencia, Spain; \\ 3 Centro de Investigación Traslacional San Alberto Magno, Universidad Católica de Valencia San Vicente \\ Mártir, 46001 Valencia, Spain; maria.escudero@ucv.es; ORCID: 0000-0001-5522-056X \\ 4 Escuela de Doctorado, Universidad Católica de Valencia San Vicente Mártir, 46008 Valencia, Spain; \\ javier.bonastre@mail.ucv.es ORCID: 0000-0003-4926-5539 \\ 5 School of Experimental Sciences, Universidad Católica de Valencia San Vicente Mártir, 46001 Valencia, \\ Spain; mario.zacares@ucv.es \\ 6 School of Medicine, Universidad Católica de Valencia San Vicente Mártir, 46001 Valencia, Spain \\ 7 Centro de Investigación Traslacional San Alberto Magno, Universidad Católica de Valencia San Vicente \\ Mártir, 46001 Valencia, Spain \\ * Correspondence: elisa.oltral@ucv.es ; ORCID 0000-0003-0598-2907
}

\begin{abstract}
Current pharmacological treatments of Fibromyalgia (FM) are merely symptom palliative, as clinical trials have so far failed to provide overall benefits without associated harms. Polypharmacy often leads to patient's health deterioration and chronic drug use to an eventual lack of patient's response. Emerging evidence support that physiotherapy treatments based on mechanical triggers improve FM symptoms and therefore could be used for therapeutic purposes by themselves, or in combination with current pharmacological treatments, as part of integrative medicine programs. However, a paucity of studies rigorously and systematically evaluating this possibility exists. This study uses scores from validated standardized questionnaires, algometer pressure point threshold (PPT) readings and responses from a custom self-developed questionnaire to determine the impact of a pressure-controlled manual protocol on FM hyperalgesia/allodynia, fatigue and patient's quality of life. The results show that patient's baseline sensitivity to pain inversely correlates with treatment response in FM. Moreover, patients presenting comorbid $\mathrm{ME} / \mathrm{CFS}$ do not seem to respond to the applied therapy as those presenting FM only. Thus, pretreatment PPTs and ME/CFS comorbidity may serve as indicators to predict patient's response to physiotherapy programs based on mechanical triggers, as the one evaluated here. These unexpected findings grant further explorations including the study of gene expression profiles associating to patient's treatment response in the blood collection of samples generated by this study.
\end{abstract}

Trial registration: ClinicalTrials.gov Identifier: NCT04174300

Keywords: Fibromyalgia; Myalgic Encephalomyelitis/Chronic Fatigue Syndrome (ME/CFS); Pressure Point Threshold (PPT); Physiotherapy; Manual Therapy (MT).

\section{Introduction}

The tenth revision of the International Classification of Diseases, Clinical Modification (ICD-10-CM) assigns the M79.7 to Fibromyalgia (FM) and defines the disease, together with fibromyositis, fibrositis and myofibrositis, as a chronic multisymptomatic disease of unknown etiology, presenting low pain threshold, muscle stiffness and tenderness, often accompanied with sleep disturbances, general fatigue, headaches and memory loss [1-4]. Similarly, Myalgic Encephalomyelitis/Chronic Fatigue 
Syndrome (ME/CFS) was classified with the code R53.82 or G93.3 if post-viral. The disease is described as an acquired, complex syndrome characterized by exercise-induced exacerbated fatigue, also known as PEM (post-exertional malaise), flu-like symptoms, sleep disturbances, cognitive dysfunction and orthostatic intolerance, in conjunction with others [5,6]. Some authors have posited that they are part of the same somatic syndrome based on observed overlapping symptoms [7-9], some analytical coincidences, like the increased lactate levels in ventricular cerebrospinal fluid reported by B. Natelson et al., [10] and epidemiologic overlaps [11]. However, a list of differences in clinical and biological parameters, including PEM and autonomic function, hormone unbalance, and differential cytokine, microRNA and gene expression profiles [12-19], suggests that the underlying pathophysiology in FM differs from that of ME/CFS [10].

Current pharmacological treatments for FM and/or CFS/ME patients are exclusively directed to palliate symptoms, since clinical trials have so far failed to provide overall benefits without associated harms [20-22]. In addition, polypharmacy often leads to patient deterioration, particularly for those suffering from chemical sensitivity; and extended drug use translates into patient desensitization and lack of drug-response. Non-pharmacological options are mainly dealing with CBT/GET (cognitive behavioral therapy by itself or combined with gradual exercise therapy). FM patients can engage in moderate to vigorous exercise; however, they often experience difficulties performing and adhering to even moderate intensity regimes because of increased pain symptoms [23]. On another side, the PACE trials design and outcomes continue under debate [24,25].

Physiotherapy-based treatments, manual therapy (MT) included, might provide exercise-like effects on treated tissues, by inducing blood flow and/or increased muscle tone, without demanding physical efforts from the patient, and therefore, contrary to GET, should not compromise patient's health.

MT involves the manipulation of soft tissues and joints to relieve pain, reduce inflammation, release muscle contractures and increase the range of motion, to restore health. Massage maneuvers include pressures, rubbing, friction, kneading, and vibrations, among other. Although different benefits are attributed to particular maneuvers, i.e. increased vagal tone by moderate pressures $[26,27]$, and the duration, frequency, treated areas, repetition and pressures are known to be key on the downstream effects, to date, MT protocols are still poorly defined.

Despite this lack of protocol rigor, a recent systematic review and meta-analysis of RCTs (randomized clinical trials) shows that MT improves pain, anxiety and depression in FM patients $[28,29]$, suggesting that physiotherapy treatments based on manual maneuvers could be used for therapeutic purposes by themselves, or in combination with current symptomatic pharmacological treatments as part of integrative medicine programs. Inspired by this idea, our group reviewed clinical and research evidence of MT-based therapeutics with a focus on the role of the differential effects of pressure-therapeutics [30], finding that medium load pressure massage (4.5 N), using a particular frequency and repetitions, as described by Dupont-Vergesteegden's group [31] can be used as an intervention to aid in the regrowth of muscle lost during immobilization and in sedentary deconditioned individuals, such as severely affected FM and/or CFS/ME patients. At the same time, and similarly to CBT and mindfulness, MT might engage patient's mind into relaxation, boosting happiness, and perhaps an immune, hormonal and neurotransmitter responses [24,32-35].

Documented with results from the clinic and those obtained from animal models and mimetic devices [30], our group developed a custom pressure-controlled MT protocol for the treatment of FM and evaluated patient's response to this therapeutic program in the context of the registered Clinical Trial NCT04174300. Outcome measures included the use of standardized instruments, pressure-point threshold (PPT) digital recordings, and scores from a custom questionnaire (CQ) created towards gathering patient's self-impressions of the treatment.

Furthermore, since we are interested in identifying the subjacent molecular mechanisms involved in MT mechano-transduction associated therapeutic effects, a biophysical process by which cells are capable of sensing their environment and of translating cues into biochemical signals [36,37], 
we used this Clinal Trial (CT) as a platform to generate a collection of blood fractions for future gene expression inquiries.

\section{Experimental Procedures}

\subsection{Study design and patient recruitment}

The study consisted on a pilot interventional non-randomized single arm Clinical Trial, registered with the ClinicalTrials.gov Identifier: NCT04174300, for the treatment of Fibromyalgia symptoms. The program included 8 sessions of MT (twice weekly on alternate days) (Figure 1). Each session consisting on a 25 -minute custom protocol including pressure maneuvers of about $4.5 \mathrm{~N}$, performed by a single operator (collegiate physiotherapist). Patient follow up continued one month after treatment withdrawal. Participating patients $(\mathrm{N}=40)$ were recruited by invitation through local patient associations. All participants fulfilled the ACR (American College of Rheumatology) 1990 and/or 2010 criteria [2,3] as referred by their local Medical Center. Comorbid ME/CFS diagnosis in compliance with Canadian and/or International ME/CFS criteria [5,6] was requested. Patient complete blood analytics within one month prior to study start were made available. Only patients not receiving hormonal therapy or suffering of previous serious diseases, including cancer, could enroll. Patients could not participate in pharmacological clinical trials or receive additional physiotherapy treatments while being enrolled and agreed to withdraw medication $12 \mathrm{~h}$ before blood draws.

All participants signed the corresponding informed consent previous admission. For symptom improvement monitoring and stability, the standardized validated instruments: FIQ (Fibromyalgia Impact Questionnaire) [38,39], MFI (Multi-Fatigue Inventory) [40], and the quality of life SF-36 questionnaire [41], in addition to a self-created CQ were used, and tender point sensitivity PPTs measured, all before start (baseline), after completion of treatments, and one month after receiving the last treatment (to estimate the washout period after MT) (Figure 1). The washout period consisted on a 30-day waiting period immediately following the 8 sessions where patients agreed not to receive any physiotherapy treatment. The study was approved by the Universidad Católica de Valencia San Vicente Martir Ethics Committee with code UCV/2018-2019/076. All personal data was anonymized in fulfillment of Spanish data protection laws.

\subsection{Intervention and Physiotherapist self-training}

The customized intervention protocol consisted on basic massage maneuvers, including rubbing, friction and kneading, executed slowly, with wide glides, while applying gentle to moderate pressure (about $4.5 \mathrm{~N}$ ) on patient's back, 25/min duration each, 2 sessions weekly for a total of 8 in 4 consecutive weeks. The targeted force was considered enough to be effective while avoiding excessive muscular stress on the patient [31]. The treated muscles were the trapezius (upper, middle and lower fibers), supraspinatus and infraspinatus, rhomboids, teres major and minor, latissimus dorsi, lumbar square, paravertebral muscles, the entire thoraco-lumbar fascia, the pyramidal and the gluteal musculature (gluteus maximus, middle and minor). The protocol was applied on the patient on prone position with his/her back and gluteal areas exposed.

Prior to study start the collegiate physiotherapist in charge of the Trial self-trained to ensure reproducible pressure maneuvers of the desired intensity $(4.5 \mathrm{~N})$ using a pressure-sensitive selfassembled artifact. The dispositive consisted on a flat soft-to-touch surface with a similar area to the body part to be treated, placed over four pressure sensors (Flexiforce, Tekscan, Canada). The training period consisted on repetitions of the protocol, until obtaining a triplicate of satisfactory pressure scores for 5 consecutive days. Only measures deviating $10 \%$ of the $4.5 \mathrm{~N}$ target force were considered 
satisfactory. Short pressure application operator self-checkups with the mentioned device preceded each patient session.

\subsection{Questionnaires}

In addition to the standard validated instruments to assess patient physical performance and emotional status, including the FIQ [38,39], MFI [40] and the SF-36 (Likert scale) [41] self-reported questionnaires, we developed an additional short tool to estimate patient's perception of their own response to treatment in the form of a custom questionnaire (CQ). The items included 5 questions (Q1-Q5) scored with a scale of -1 to 1 , with a -1 value if they felt worse, 0 if they felt no appreciable change or +1 if they sensed improvement right after receiving the treatment or $24 \mathrm{~h}$ after of the treated area, and mobility, pain, or fatigue. In addition an overall satisfaction question (Q6) using a scale of $1-10$, with the highest value corresponding to highest level of satisfaction, was included to estimate patient's overall assessment of the treatment. The formulated translated questions were:

- Q1-How did you perceived the treated region right after each treatment?

- Q2- How did you perceived the treated region $24 \mathrm{~h}$ after each treatment?

- Q3- How is the mobility of your back after the full 8-session treatment?

- Q4-How is your back (sensation and pain) after the full 8-session treatment? (comparing before and after)

- Q5-How do you feel at the level of fatigue after the full 8-session treatment? (comparing before and after)

- Q6-How do you find the effectiveness of the treatment after its completion? (full 8-session treatment)

The CQ instrument was also used to evaluate patient's perception on the stability of the treatment one month after the treatment ended. Q1-was reformulated to: How do you perceive the treated region now in comparison to the feeling right after treatment? Q2 was ommited, and Q6 was intended to assess the washout period for those finding the treatment effective (responders).

\subsection{Pressure Point Threshold Assesment}

To assess Pressure Pain Thresholds (PPTs) of tender point sites in patients with FM, we used the FDIX Force Gage, ForceOne algometer (Wagner Instruments, USA) with a capacity of $200 \mathrm{lbf} / 100 \mathrm{kgf}$ $/ 1000 \mathrm{~N}$ and with an accuracy of $\pm 0.2 \%$ dedicated (single force) and $\pm 0.3 \%$ interchangeable (multiple force) cell modules. A $1 \mathrm{~cm} 2$ rubber disk was used to press on the points to be evaluated using a $90^{\circ}$ angle. The values were registered in lbf.

PPTs were measured for each of the 18 tender points located in the muscles/areas indicated for the diagnosis of FM. Bilaterally, they include: occiput, trapezius, supraspinatus, gluteal, low cervical , second rib, lateral epicondyle humerus, greater trochanter and knee. The patient was placed in a supine position for the assessment of the following PPTs: low cervical, second rib, lateral epicondyle humerus, greater trochanter and knees; while for the assessment of the remaining PPTs (occiput, trapezius, supraespinatus and gluteal), the patient was placed in the prone position. The anatomic location of the pain points was appraised by the evaluating physiotherapist through palpation. The placement of the algometer was perpendicular to the point where the constant pressure was to be applied to assess the sensitivity of the patient. Each sampling consisted of 3 independent pressure measurements, allowing a recovery time of 10 seconds between each one. Before pressure tests started, patients were instructed to indicate when the pressure change caused pain, as previously described [42,43].

\subsection{Blood collection}

A collection of blood fractions was generated and registered at the Institute of Health Carlos III National Biobank, Madrid, Spain with the intention to evidence molecular changes induced by this pressure-controlled custom physiotherapy treatment and its stability. The collection consisted on 
plasma and PBMCs (peripheral blood mononuclear cells) obtained at baseline (before treatment started), after the first therapeutic session, after the $4^{\text {th }}$ therapeutic session, after completing the 8session treatment and after 30-day wait period). Twenty $\mathrm{ml}$ aliquots were drawn by a collegiate nurse, using sodium citrate as anticoagulant (BD tubes, cat. 367691). Within $4 \mathrm{~h}$ after blood draw the samples were centrifuged at $1500 \mathrm{rpm}(453 \mathrm{~g})$ during $15 \mathrm{~min}$ on a table preparative centrifuge. Plasma (approximate 8-12 $\mathrm{mls}$ ) were transferred to 2-ml Eppendorf tubes and centrifuged to remove contaminating cells and platelets at $10,000 \mathrm{~g}$ for $10 \mathrm{~min}$ on a microcentrifuge. Supernatants were aliquoted into 1-ml cryovials. PBMCs were obtained from the rest of the blood, after removing the plasma, by dilution in phosphate-buffered saline solution (PBS) at 1:1 (v/v) ratio of the original volume, layering over one volume of Ficoll-Paque Premium (GE Healthcare, Chicago, IL, USA) followed by density centrifugation separation at $500 \mathrm{~g}$ for $30 \mathrm{~min}\left(20^{\circ} \mathrm{C}\right.$, brakes off). The PBMC layer was washed with PBS and resuspended in red blood cell lysis buffer $(155 \mathrm{mM} \mathrm{NH} 4 \mathrm{Cl}, 10 \mathrm{mM}$ NaHCO3, $0.1 \mathrm{mM}$ EDTA, and $\mathrm{pH} 7.4)$, kept on ice for $5 \mathrm{~min}$, and centrifuged $\left(20{ }^{\circ} \mathrm{C}\right.$ at $500 \mathrm{~g}$ for 10 $\mathrm{min})$, to remove contaminating erythrocytes. Washed pellets were snap frozen after removal of supernatant. Plasma aliquots were preserved at $-80^{\circ} \mathrm{C}$ and PBMCs at $-150^{\circ} \mathrm{C}$ until further use, as previously described $[18,44]$.

Registration of the blood collection was done at the National Spanish Biobank Network (National Institute Carlos III) by completing the CT and collection details digitally on the Biobank Network website https://biobancos.isciii.es/NuevaColeccion.aspx

\subsection{Statistical Analysis and Plotting}

Continuous data are expressed as means \pm SD and range as indicated. Statistical differences were determined using two-tailed unpaired $t$-tests for normal values and either Mann Whitnney or Wilcoxon nonparametric test if the values were found no to follow a normal distribution. Values normal distribution was assessed by the Shapiro-Wilk normality test. Differences between groups were considered significant when $p \leq 0.05$. Analysis were conducted with Excel and with the SPSS package 13.0 (SPSS Inc, Chicago, IL, USA) and R 3.6.1 [45]. Variable correlations were evaluated by the simple linear regression method (least-squares approach). Plots were drawn using the GraphPad Prism 5.0 program (San Diego, CA, USA) and the package ggplot2 [46].

\section{Results}

\subsection{Study design}

The Clinical Trial NCT04174300 consisted on a pressure-controlled custom physiotherapy treatment of 10 out of the $18 \mathrm{FM}$ tender points and surrounding areas, following a study design as outlined on Figure 1 and detailed on the Experimental Procedures section, towards triggering painreducing mechanisms. Here we show the results obtained for the outcome variables measuring pain, fatigue and quality of life, using standard validated instruments and PPT scores as an approach to evidence response to treatment. In addition, a CQ was added to the FIQ, MFI and SF-36 instruments and algometer PPTs, which was used only after completing the 8-session treatment and after a 30day washout period, consisting on a waiting time not receiving manual therapy to determine the stability of therapy-induced improvements. The trial included blood draws at different time points, as detailed in the Flowchart below and the Methods section. The approximately 200 sample collection derived from 40 individuals donating blood at 5 time points [pre-treatment, after the first session, after completing the $4^{\text {th }}$ session, after completing the 8 -session procedure and 30 -day after completing the 8-sessions as a waiting period without treatment (washout period)], yielded about 1600 aliquots (800 of plasma and 800 corresponding to PBMCs). This collection of samples was registered at the Institute of Health Carlos III National Biobank Network, Madrid, Spain with collection number C.0006173, with the intention to evidence molecular changes induced by this pressure-controlled custom physiotherapy treatment in future studies. 


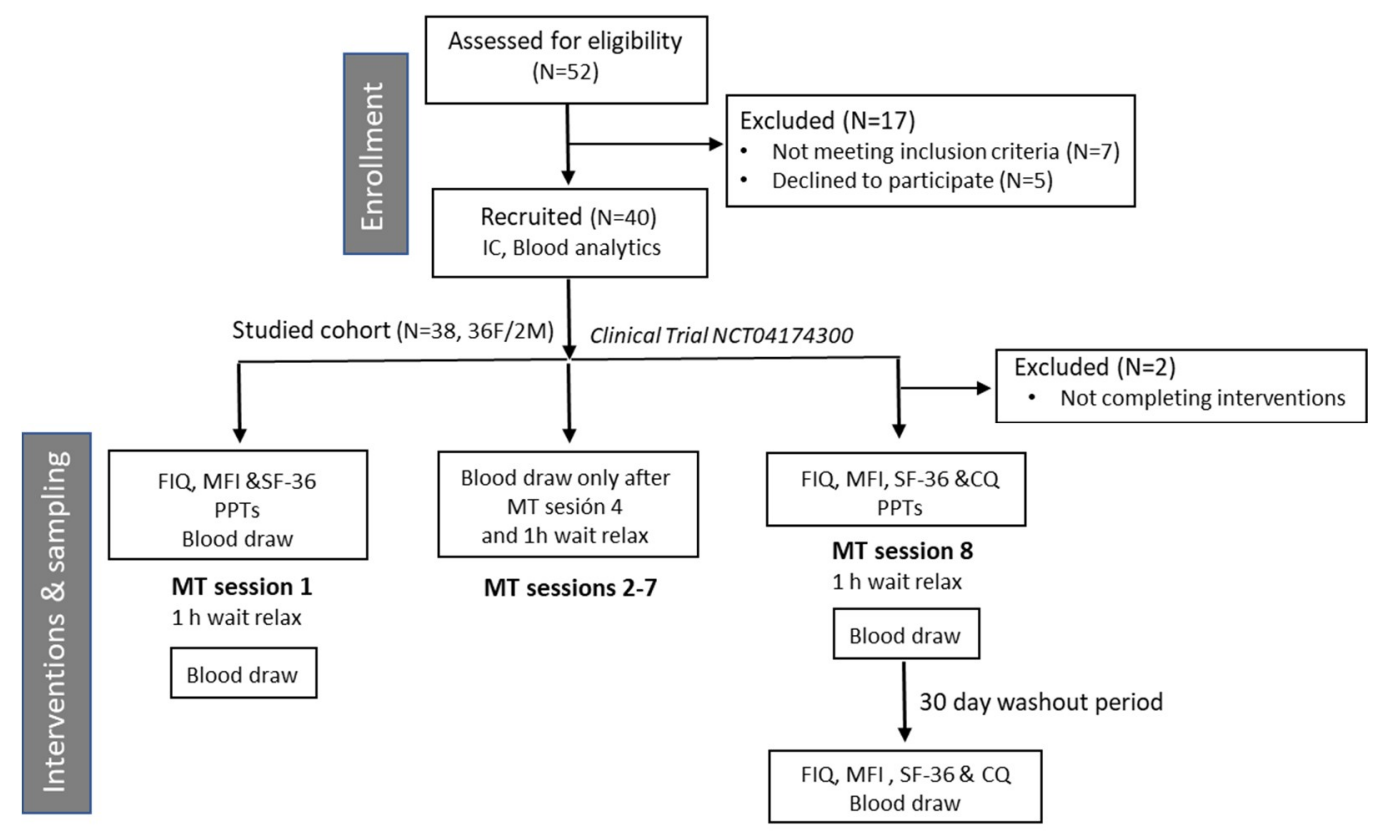

Figure 1. Flowchart depicting enrollment, intervention and sampling steps of the NCT04174300. IC (Informed Consent). FIQ (Fibromyalgia Impact Questionnaire), MFI (Multi Fatigue Inventory), SF36 quality of life questionnaire, CQ (Custom Questionnaire). PPTs (Pressure Point Threshold). MT (Manual Therapy).

\subsection{Cohort demographics}

The final studied cohort included $38 \mathrm{FM}$ patients (35 females and 3 males) who fulfilled 1990 and/or 2010 ACR criteria [2,3], with 50\% (19/38) of them presenting comorbid ME/CFS according to Canadian and/or International diagnostic criteria $[5,6]$. Only one of the three participating males fulfilled ME/CFS criteria. Two of the initially 40 recruited patients had to be excluded for not completing the treatment, due to reasons unrelated to the therapy. Average age was $55.6 \pm 7.2$ years (range 43-71) and time from primary FM diagnosis over 3 years, $10.3 \pm 7.5$ years (range 3-21).

\subsection{Patient characteristics}

\subsubsection{Patient health status at baseline}

As determined with the indicated standard questionnaires (please see the Experimental Procedures section for details), total FM impact score of the studied cohort at baseline was $72.62 \pm$ 15.67, range (41.08-96.26); general fatigue at the beginning of the study was $11.5 \pm 1.62$, range (7-16), which included physical and mental fatigue; and the perceived quality of life at recruitment was poor according to SF-36 scores, affecting physical, mental and emotional roles of participants (Table 1). According to the total FIQ scores of participants and taking into accout that a score $<39$ indicates mild affection, $\geq 39$ through $\leq 59$ is assigned to moderately affected patients, and $>59$ to severe; the studied cohort can be described as mainly severe, with 79\% (30/38) of the participants presenting severe FM and only $21 \%(8 / 38)$ moderate affection. Supplementary Table S1 shows itemized findings.

Table 1. Patient health status assessment with the FIQ, MFI and SF-36 instruments [38-41] at baseline $(\mathrm{N}=38)$. 


\begin{tabular}{lccc}
\hline \multicolumn{1}{c}{ Questionnaire } & Mean & SD & Range \\
\hline \multicolumn{1}{c}{ FIQ } & & & \\
\hline Total FIQ & 72.62 & 15.67 & {$[41.08-96.26]$} \\
Function & 5.16 & 2.29 & {$[0-9.24]$} \\
Overall & 8.3 & 2.29 & {$[2.86-10.01]$} \\
Symptoms & 4.59 & 2.23 & {$[0-10.01]$} \\
\hline \multicolumn{1}{c}{ MFI } & & & \\
\hline General Fatigue & 11.5 & 1.62 & {$[7-16]$} \\
Physical Fatigue & 12.3 & 1.23 & {$[16-10]$} \\
Reduced Activity & 12.1 & 1.93 & {$[6-19]$} \\
Reduced Motivation & 10.6 & 2.72 & {$[7-15]$} \\
Mental Fatigue & 11.5 & 1.75 & {$[7-15]$} \\
\hline \multicolumn{1}{c}{ SF-36 } & & & \\
\hline Physical Functioning (PF) & 38.95 & 17.48 & {$[0-85]$} \\
Role Physical (RP) & 28.95 & 21.87 & {$[0-81.25]$} \\
Bodily Pain (BP) & 26.64 & 18.39 & {$[0-70]$} \\
General Health (GH) & 29.68 & 16.03 & {$[0-65]$} \\
Vitality (VT) & 16.12 & 15.83 & {$[0-50]$} \\
Social Functioning (SF) & 35.2 & 27.39 & {$[0-87.5]$} \\
Role Emotional (RE) & 56.58 & 37.12 & {$[0-100]$} \\
Mental Health (MH) & 47.24 & 21.92 & {$[5-90]$} \\
\hline
\end{tabular}

FIQ (Fibromyalgia Impact Questionnaire), MFI (Multi Fatigue Inventory) and SF-36 quality of life questionnaire. SD (standard deviation). Range refers to the possible values in the studied group.

\subsubsection{Blood analytics of participants}

Blood analytics of participants did not show consistent alterations in any of the measured variables. However, it was interesting to observe that some patients presented off-range values for some parameters. In particular, it seemed noticeble that $63.8 \%(23 / 36)$ participants presented low percentage of neutrophils, according to normal range (Supplementary Table S1). Other blood parameters that deviated from established normal reference values were: the percentage of monocytes, which appeared increased in $41.6 \%$ (15/36) of the study participants; and the high cholesterol $(72.2 \%$ or $26 / 36)$ and low LDL (40\% or 13/32). Mild hipercholesteremia in an advanced age group is considered usual and unrelated to the FM phenotype. An interesting, less prevalent subgroup, was that constituted by $6 / 36$ participants $(16.7 \%)$ with a higher than normal percentage of lymphocytes that could be indicative of an infectious subgroup. However, only one of the 6 presented clear higher absolute counts (Supplementary Table S2). A higher percentage of them (22.9\% or 8/35) presented higher levels of creatinine, which could relate to muscle damage. It should be noted that no blood analytics were available for 2 of the study participants and not all the variables were assayed for the complete group.

\subsubsection{Tender point sensitivity at baseline}

To estimate tender point sensitivity of participants (hyperalgesia/allodynia) each of the 18 anatomically sensitive areas described in FM [2] were localized and measured by triplicate with a FDIX Force Gage, ForceOne algometer (Wagner Instruments, USA) as detailed in Experimental Procedures. The average PPT values for each point, together with standard deviations and value range, are shown on Table 2. Scores show that the Low cervical points were the most sensitive while Gluteus, Trochanters and Knees were the most resistant to pain (Table 2). Supplementary Table S3 shows PPT average for individual recordings. Ten out of the 18 PPTs were treated with our physiotherapy program while 8 of them were not, as indicated with asterisks on Table 2 .

Table 2. Patient tender point sensitivity assessment, as determined by triplicate measurements in $\mathrm{lbf}$ with a FDIX Force Gage, ForceOne algometer (Wagner Instruments, USA) at baseline ( $\mathrm{N}=38$ ). 


\begin{tabular}{lcccccc}
\hline \multicolumn{7}{c}{ Lbf (pound force) } \\
\hline \multicolumn{1}{c}{ Tender points } & Mean PPTs pre- & Mean PPTs post- & SD pre- & SD post- & p-value & Range \\
\hline Occiput. right* & 0.8062 & 0.8404 & 0.3775 & 0.3187 & 0.3421 & {$[0.065-1.525]$} \\
Occiput. left* & 0.8606 & 0.8095 & 0.4152 & 0.3264 & 0.3281 & {$[0.097-1.733]$} \\
Trapezius. right* & 0.9371 & 1.0741 & 0.3521 & 0.3597 & $\mathbf{0 . 0 0 7 5}$ & {$[0.240-1.493]$} \\
Trapezius. left* & 0.9757 & 1.1075 & 0.4106 & 0.3955 & $\mathbf{0 . 0 2 5 0}$ & {$[0.140-1.825]$} \\
Supraspinatus. right* & 1.0115 & 1.0855 & 0.4311 & 0.4139 & 0.2168 & {$[0.217-1.905]$} \\
Supraspinatus. left* & 1.0050 & 1.0794 & 0.4045 & 0.3470 & 0.2275 & {$[0.177-1.838]$} \\
Gluteal. right* & 1.3336 & 1.5417 & 0.6371 & 0.5877 & $\mathbf{0 . 0 1 4 2}$ & {$[0.158-2.780]$} \\
Gluteal. left* & 1.3617 & 1.5256 & 0.6375 & 0.6178 & $\mathbf{0 . 0 2 4 3}$ & {$[0.207-2.670]$} \\
Low cervical. right* & 0.4879 & 0.3875 & 0.2669 & 0.1565 & $\mathbf{0 . 0 1 1 5}$ & {$[0.000-1.172]$} \\
Low cervical. left* & 0.4732 & 0.3748 & 0.2384 & 0.1235 & $\mathbf{0 . 0 1 0 2}$ & {$[0.095-1.070]$} \\
Second rib. right & 0.7123 & 0.5764 & 0.4741 & 0.2492 & $\mathbf{0 . 0 4 3 3}$ & {$[0.152-2.665]$} \\
Second rib. left & 0.7036 & 0.6348 & 0.4567 & 0.2691 & 0.3015 & {$[0.153-2.307]$} \\
Lateral Epicondyle humerus. right & 0.8170 & 0.7335 & 0.4332 & 0.2781 & 0.2208 & {$[0.080-1.830]$} \\
Lateral Epicondyle humerus. left & 0.8495 & 0.7501 & 0.3602 & 0.3054 & 0.0744 & {$[0.298-1.823]$} \\
Greater trochanter. right & 1.9234 & 1.7825 & 0.9234 & 0.8721 & 0.1181 & {$[0.285-3.892]$} \\
Greater trochanter. left & 1.8306 & 1.8472 & 0.8634 & 0.8665 & 0.8974 & {$[0.472-3.955]$} \\
Knee. right & 1.1938 & 1.1096 & 0.6087 & 0.3921 & 0.2799 & {$[0.263-2.505]$} \\
Knee. left & 1.2958 & 1.1464 & 0.7362 & 0.4644 & 0.0785 & {$[0.000-2.980]$} \\
\hline
\end{tabular}

$\left(^{*}\right)$ Tender points in areas treated with manual therapy; PPT (Pressure Point Threashold); SD (Standard Deviation); pre- (pre-treatment); post- (post-treatment. Significant differences $(p \leq 0.05)$ are bolded.

\subsection{Patient response to therapy according to questionnaires}

The instruments used to evaluate pain, fatigue and quality of life at baseline (before the MT treatment started), were likewise used to assess changes in these parameters after completion of the 8-session treatment and also one month after the treatment ended, with the aim of estimating washout period of the response to treatment. As previously mentioned, patients agreed not to receive any physiotherapy session during the 30-day waiting period.

\subsubsection{Response to treatment of FM patients with or without comorbid ME/CFS}

As shown on Table 3, and detailed on Supplementary Table S1, only Total FIQ, FIQ Overall and SF-36 BP showed significant differences when scores after the physiotherapy treatment were compared to baselines' [p-values (1)]. Interestingly, differences for both FIQ total and FIQ Overall were also significant when the Pre-treatment and the Post-washout treatment were compared [pvalues 2)], indicating that the benefits obtained from the therapy, were lasting over a 30-day washout period. By contrast, SF-36 BP difference was not kept after this same wait period suggesting that although some general benefits persisted, the improvement in pain may be lost when therapy is discontinued for the indicated period of time.

Table 3. Patient response to therapy as evidenced by score differences in the standard, validated, FIQ, MFI and SF-36 instruments [38-41]. 


\begin{tabular}{lcccccc}
\hline \multicolumn{1}{c}{ Questionnaire } & Pre-treatment & Post-treatment & Post-washout & p-value (1) & p-value (2) & p-value (3) \\
\hline \multicolumn{1}{c}{ FIQ } & & & & & & 0.9370 \\
\hline Total FIQ & $72.62 \pm 15.67$ & $64.15 \pm 18.25$ & $63.82 \pm 18.30$ & $\mathbf{0 . 0 3 3 3}$ & $\mathbf{0 . 0 2 7 4}$ & 0.9877 \\
Function & $5.16 \pm 2.29$ & $4.62 \pm 2.43$ & $4.61 \pm 2.48$ & 0.3240 & 0.3209 & 0.8476 \\
Overall & $8.30 \pm 2.23$ & $6.74 \pm 3.05$ & $6.96 \pm 2.79$ & $\mathbf{0 . 0 1 2 2}$ & $\mathbf{0 . 0 1 1 7}$ & 0.2921 \\
Symptoms & $4.59 \pm 3.72$ & $4.14 \pm 3.32$ & $3.39 \pm 3.14$ & 0.7518 & 0.2139 & \\
\hline \multicolumn{1}{c}{ MFI } & & & & & 0.3055 \\
\hline General Fatigue & $11.5 \pm 1.6$ & $11.7 \pm 1.1$ & $11.9 \pm 1.7$ & 0.6823 & 0.4383 & 0.6592 \\
Physical Fatigue & $12.3 \pm 1.2$ & $12.4 \pm 1.8$ & $12.4 \pm 2.1$ & 0.9092 & 0.8124 & 0.3838 \\
Reduced Activity & $12.1 \pm 1.9$ & $12.2 \pm 2.3$ & $12.4 \pm 1.8$ & 0.5709 & 0.8507 & 0.3940 \\
Reduced Motivation & $10.6 \pm 2.7$ & $10.7 \pm 2.6$ & $11.0 \pm 2.2$ & 0.3940 & 0.8765 & 0.9058 \\
Mental Fatigue & $11.5 \pm 1.8$ & $11.9 \pm 1.7$ & $11.6 \pm 1.6$ & 0.3972 & 0.4786 & \\
\hline \multicolumn{1}{c}{ SF-36 } & & & & & & 0.4887 \\
\hline Physical Functioning (PF) & $38.95 \pm 17.48$ & $41.46 \pm 16.55$ & $41.71 \pm 17.14$ & 0.5218 & 0.9486 & 0.3441 \\
Role Physical (RP) & $28.95 \pm 21.87$ & $34.21 \pm 25.24$ & $32.40 \pm 21.89$ & 0.3419 & 0.7732 & 0.5879 \\
Bodily Pain (BP) & $26.64 \pm 18.39$ & $36.45 \pm 23.65$ & $32.11 \pm 24.86$ & $\mathbf{0 . 0 4 7 3}$ & 0.2341 & 0.4654 \\
General Health (GH) & $29.68 \pm 16.03$ & $27.76 \pm 15.14$ & $27.11 \pm 14.64$ & 0.3763 & 0.9010 & 0.9249 \\
Vitality (VT) & $16.12 \pm 15.83$ & $20.53 \pm 21.71$ & $16.12 \pm 16.09$ & 0.6752 & 0.5948 & 0.1004 \\
Social Functioning (SF) & $35.20 \pm 27.39$ & $46.91 \pm 27.20$ & $45.72 \pm 28.81$ & 0.0563 & 0.8543 & 0.5395 \\
Role Emotional (RE) & $56.58 \pm 37.12$ & $53.51 \pm 34.64$ & $61.84 \pm 35.49$ & 0.6599 & 0.3037 & 0.2120 \\
Mental Health (MH) & $47.24 \pm 21.92$ & $54.08 \pm 22.08$ & $53.95 \pm 24.47$ & 0.1794 & 0.9804 & \\
\hline
\end{tabular}

FIQ (Fibromyalgia Impact Questionnaire), MFI (Multi Fatigue Inventory) and SF-36 quality of life questionnaire. SD (standard deviation). Range refers to the possible values in the studied group. P-value (1) refers to the pvalues obtained when the groups compared were the Pre- and the Post-treatment; p-value (2) refers to the pvalues obtained for comparison of the Pre- and the Post-washout setd of data; and p-value (3) to pvalues corresponding to differences between groups Post-treatment and Post-washout. Significant differences $(\mathrm{p}<0.05)$ appear in bold.

When the criteria to assess the impact of the treatment on patient symptoms was our $C Q$, the results further supported that the 30-day wash period was enough to loose treatment benefits according to the significant differences found for the Response to treatment and the Total questionnaire scores, with lower means in the Post-washout (Table 4). Interestingly enough, patients perceived that the improvement or response to therapy was higher immediately after the treatment $(0.97 \pm 0.16)$ that $24 \mathrm{~h}$ after $(0.39 \pm 0.79)$. They also perceived no major benefits from the treatment to alleviate their fatigue.

Table 4. Patient response to therapy assessed by score-differences in our CQ

\begin{tabular}{lccccc}
\hline \multicolumn{1}{c}{ Custom Questionnaire } & $\begin{array}{c}\text { Post- } \\
\text { treatment }\end{array}$ & $\begin{array}{c}\text { Range Post- } \\
\text { treatment }\end{array}$ & $\begin{array}{c}\text { Post- } \\
\text { washout }\end{array}$ & $\begin{array}{c}\text { Range Post- } \\
\text { washout }\end{array}$ & p-value \\
\hline Response to treatment & $0.97 \pm 0.16$ & {$[0-1]$} & $0.71 \pm 0.61$ & {$[-1-1]$} & $\mathbf{0 . 0 1 9 7}$ \\
Response to treatment after $24 \mathrm{~h}$ & $0.39 \pm 0.79$ & {$[-1-1]$} & $\mathrm{N} / \mathrm{A}$ & $\mathrm{N} / \mathrm{A}$ & $\mathrm{N} / \mathrm{A}$ \\
Improves mobility & $0.87 C 0.34$ & {$[0-1]$} & $0.68 \pm 0.47$ & {$[0-1]$} & $\underline{0.0969}$ \\
Pain reponse & $0.82 \pm 0.46$ & {$[-1-1]$} & $0.66 \pm 0.48$ & {$[0-1]$} & $\underline{0.1106}$ \\
Reponse to fatigue & $0.24 \pm 0.54$ & {$[-1-1]$} & $0.24 \pm 0.49$ & {$[-1-1]$} & $>0.9999$ \\
Final treatment effectiveness & $7.68 \pm 2.44$ & {$[0-10]$} & $6.88 \pm 2.68$ & {$[0-10]$} & 0.1558 \\
Total questionnaire & $10.97 \pm 3.59$ & {$[-1-15]$} & $9.17 \pm 3.76$ & {$[0-14]$} & $\mathbf{0 . 0 0 8 5}$ \\
\hline
\end{tabular}

N/A: not available. Significant differences $(\mathrm{p}<0.05)$ appear in bold. Tendencies $(\mathrm{p} \leq 0.1)$ are undelined.

\subsubsection{Response to treatment of patients with FM only vs FM patients with comorbid ME/CFS}

Since our cohort included $50 \%$ of the patients diagnosed for FM only, and 50\% diagnosed with comorbid ME/CFS, we decided to explore whether the initial health status of the patient could play a role on the response to therapy, particularly since a differential physiopatology has been attributed to these overlapping diseases [10]. For this purpose we reassessed the statistical analysis performed 
after stratification of the patients in two groups ( $\mathrm{N}=19 / \mathrm{each})$ acording to having received diagnosis for one or the other disease by a collegiate clinician, as detailed in Methods.

It was interesting to find that several items from the questionnares, particularly the FIQ and the SF-36, clearly differentiated these subgroups of patients. For example, Total FIQ and Overall showed differences between groups of patients before and after treatment, either with a tendency $(p<0.1)$, or with statistical significance ( $\mathrm{p} \leq 0.05)$. The significant FIQ differences after treatment seem to indicate preferential response in the FM only subgroup (please compare pre- and post- means for each subgroup). This appears to be also the case for the SF-36 items: Vitality (VT), Social Functioning (SF) and Bodily Pain (BP). It is also interesting to note that the SF-36 Mental Health (MH) item which did not show differences between subgroups at the start of the study (Pre-treatment), did show differences in the Post-treatment and even in the Post-washout period values, with means in the FM group that clearly showed scores for improvement. Therefore, the benefits of the therapy seem to favor the FM subgroup for both pain and functioning, social activities and mental health (Table 5).

Table 5. Differences between FM patients presenting or not comorbid ME/CFS, assessed by FIQ, MFI and SF-36 instruments [38-41].

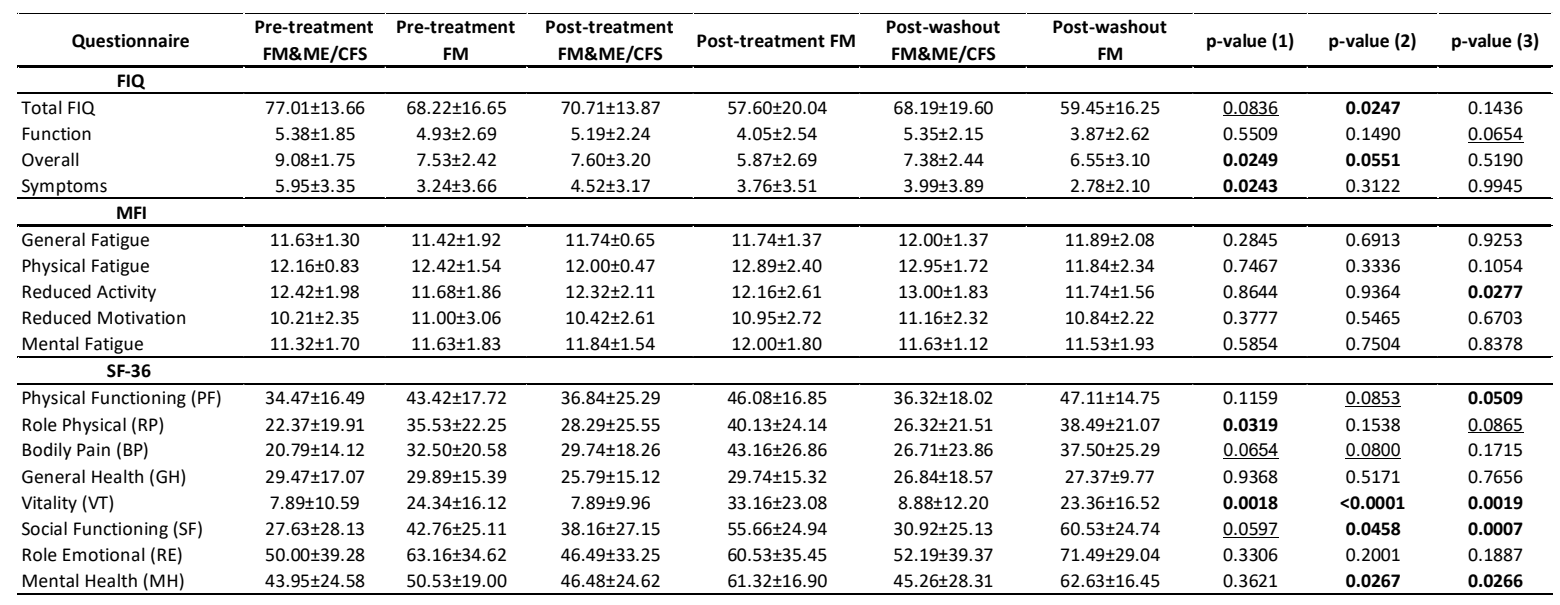

FIQ (Fibromyalgia Impact Questionnaire), MFI (Multi Fatigue Inventory) and SF-36 quality of life questionnaire. FM (Fibromyalgia only), FM\&ME/CFS (Fibromyalgia with comorbid ME/CFS). P-value (1) refers to the pvalues obtained when the groups compared were the Pre-treatment FM\&ME/CFS and the Pre-treatment FM; p-value (2) refers to the pvalues obtained for comparison of the Post-treatment FM\&ME/CFS and the Posttreatment FM sets of data; and p-value (3) to pvalues corresponding to differences between groups Postwashout FM\&ME/CFS and the Post-washout FM. Significant differences $(\mathrm{p} \leq 0.05)$ appear in bold. Tendencies $(\mathrm{p}<0.1)$ are undelined.

When the subgroups of patients were individually analyzed for patient response to therapy, significant differences could be confirmed in the FM only subgroup for the SF and MH, in addition to some tendencies in the Total FIQ and Overall (Supplementary Table S4). The variability of the scores and low number of participants after stratification may hamper the identification of additional significant changes. By contrast, the FM\&ME/CFS subgroup did not show improvements other that the FIQ overall when the pre- and the post-washout conditions were compared, and only tendencies in MFI Physical fatigue and Reduced activity in Post-washout sets of data (Supplementary Table S5), perhaps indicating a delayed benefit of the therapy. Larger group analysis may be necessary to definitively determine symptom changes with these instruments.

Similarly, our CQ further supportes preferential improvement of the FM subgroup with respect to the FM\&ME/CFS (Tables 6 and 7), highlighting pain response and mobility over fatigue.

Table 6. FM only patient subgroup response to therapy, assessed by score-differences in our CQ. 


\begin{tabular}{lccccc}
\hline \multicolumn{1}{c}{ Questionnaire } & $\begin{array}{c}\text { Post- } \\
\text { treatment }\end{array}$ & $\begin{array}{c}\text { Range Post- } \\
\text { treatment }\end{array}$ & $\begin{array}{c}\text { Post- } \\
\text { washout }\end{array}$ & $\begin{array}{c}\text { Range Post- } \\
\text { washout }\end{array}$ & p-value \\
\hline Response to treatment & $1.00 \pm 0.00$ & {$[1-1]$} & $0.79 \pm 0.54$ & {$[-1-1]$} & 0.2297 \\
Response to treatment after $24 \mathrm{~h}$ & $0.42 \pm 0.84$ & {$[-1-1]$} & $\mathrm{NA}$ & $\mathrm{NA}$ & $\mathrm{NA}$ \\
Improves mobility & $1.00 \pm 0.00$ & {$[1-1]$} & $0.74 \pm 0.45$ & {$[0-1]$} & $\mathbf{0 . 0 4 6 3}$ \\
Pain reponse & $0.95 \pm 0.23$ & {$[0-1]$} & $0.58 \pm 0.51$ & {$[0-1]$} & $\mathbf{0 . 0 1 8 8}$ \\
Reponse to fatigue & $0.37 \pm 0.60$ & {$[-1-1]$} & $0.37 \pm 0.60$ & {$[-1-1]$} & $>0.9999$ \\
Final treatment effectiveness & $8.19 \pm 1.77$ & {$[3-10]$} & $7.26 \pm 2.05$ & {$[3-10]$} & 0.1814 \\
Total questionnaire & $11.89 \pm 2.38$ & {$[5-15]$} & $9.74 \pm 3.09$ & {$[3-14]$} & $\mathbf{0 . 0 2 5 6}$ \\
\hline
\end{tabular}

N/A: not available. Significant differences $(\mathrm{p}<0.05)$ appear in bold.

Table 7. FM\&ME/CFS patient subgroup response to therapy, assessed by score-differences in our CQ.

\begin{tabular}{lccccc}
\hline \multicolumn{1}{c}{ Questionnaire } & $\begin{array}{c}\text { Post- } \\
\text { treatment }\end{array}$ & $\begin{array}{c}\text { Range Post- } \\
\text { treatment }\end{array}$ & $\begin{array}{c}\text { Post- } \\
\text { washout }\end{array}$ & $\begin{array}{c}\text { Range Post- } \\
\text { washout }\end{array}$ & p-value \\
\hline Response to treatment & $0.95 \pm 0.23$ & {$[0-1]$} & $0.63 \pm 0.68$ & {$[-1-1]$} & 0.1264 \\
Response to treatment after $24 \mathrm{~h}$ & $0.37 \pm 0.76$ & {$[-1-1]$} & $\mathrm{NA}$ & $\mathrm{NA}$ & $\mathrm{NA}$ \\
Improves mobility & $0.74 \pm 0.45$ & {$[0-1]$} & $0.63 \pm 0.50$ & {$[0-1]$} & 0.7281 \\
Pain reponse & $0.68 \pm 0.58$ & {$[-1-1]$} & $0.74 \pm 0.45$ & {$[0-1]$} & $>0,9999$ \\
Reponse to fatigue & $0.11 \pm 0.46$ & {$[-1-1]$} & $0.11 \pm 0.32$ & {$[0-1]$} & $>0,9999$ \\
Final treatment effectiveness & $7.21 \pm 2.94$ & {$[0-10]$} & $6.50 \pm 3.21$ & {$[0-10]$} & 0.5045 \\
Total questionnaire & $10.05 \pm 4.36$ & {$[-1-14]$} & $8.61 \pm 4.34$ & {$[0-13]$} & 0.2275 \\
\hline
\end{tabular}

N/A: not available. Significant differences $(\mathrm{p}<0.05)$ appear in bold.

\subsection{Patient response to therapy according to PPTs}

\subsubsection{Response to treatment of FM patients with or without comorbid ME/CFS}

As shown on Table 2, out of the five treated anatomic regions (Occiput, Trapezius, Supraspinatus, Gluteal, Low cervical) four tender points became less sensitive to pain pressure after the 8-session MT treatment, indicating overall patient's improvement in the treated area. In particular, Trapezius and Gluteal significantly improved on both sides, while Occiput and Supraspinatus did not reach significance. Unexpectedly, the most sensitive to pain tender point among the treated: the Low cervical, increased pain sensitivity on both sides, suggesting a negative effect of the treatment in this region. PPTs of untreated areas, including the tender points: Second rib, Lateral epicondyle humerus, Greater trochanter and Knee, appeared unchanged (Table 2), with the exception of the right Second rib which sensitivity significantly increased after treatment. The significance of this change, if any, is presently unknown.

Interestingly enough, negative linear correlations between the initial PPT values and the differences or changes after treatment were observed for all 18 PPTs. This suggests that the initial status of the patient, with respect to pain perception, could be indicative of the patient's capacity of response to the applied physiotherapy treatment. The findings show that in general patients with lower PPT scores (increased pain sensitivity) were capable of an increased benefit (highest difference or gained pain resistance after treatment, or change post-pre), while for those more resistant to pressure-induced pain (higher pre-treatment PPT values), therapy-induced benefits would be minor or absent (Figure 2).

Best R2 coefficients or goodness of the linear fit, on both body sides, corresponded to PPT values for the Low cervicals within the sets of treated PPTs, and to the Knee's for the non-treated PPTs, as shown on Figure 2. These points at the sensitive Low cervical PPTs and the Knee PPTs may consitute potential predictors for the patient response to MT therapy. Moreover, the distribution of values shows that although mean values seem to indicate worsening of this highly sensitive tender point (Low cervical) by treatment, there is a subgroup with low PPT baseline values that obtain a benefit (Post-pre PPT difference value $>0$ on Figure 2). 
Individual plots for the assessment of PPT-dependent patient response for the remaining 14 tender points are available on Supplementary Figure S1. Improved R2 values with respect to the Knee's were obtained for the linear models of Second rib PPTs. However, the narrow distribution of the PPT values may lead to drastic changes in additional explorations, suggesting that the Knee PPTs constitute a better option among the non-treated areas to predict patient's response to therapy.
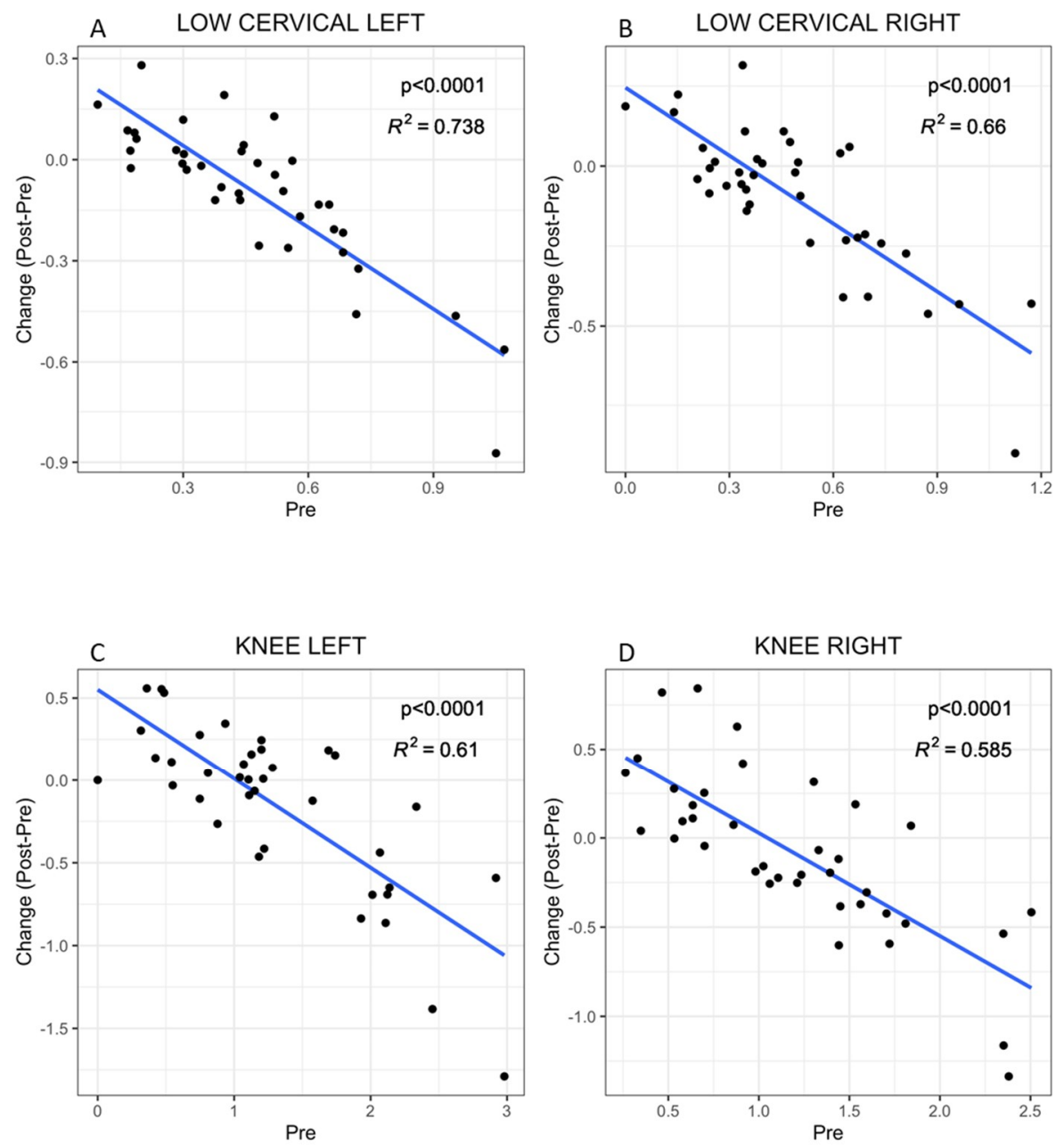

Figure 2. Linear dependence of PPT baseline scores with MT therapy response. The plots show the inverse correlation between pre-treatment PPT values and the improvement measured as the acquired resistance to pressure-induced pain for the tenderpoints (Change: Post-Pre) at the Low cervical left side of the body (A), or the right (B); and the same type of correlation for the tenderpoint at the left knee (C) or right knee (D). P and R2 values are shown.

\subsubsection{Response to treatment of patients with FM only vs FM patients with comorbid ME/CFS}

Since patients diagnosed with FM only seemed to respond to physiotherapy more readily than patients with FM presenting comorbid ME/CFS (FM\&ME/CFS), according to standard and our CQ questionnaires, it was of interest to reevaluate patients response to therapy by PPT assessment in either subgroup.

Interestingly enough, the results of these analysis show that opposite to the FM subgroup, PPTs were not good predictors of response to therapy in patients presenting comorbid ME/CFS (Figure 3 and Supplementary Figure S2). In fact, patients with both, FM and ME/CFS showed poor or no response, independent of their sensitivity threshold to pain, for some tender points, as clearly shown on Figure 3 B-D. Importantly, 5 out of the 10 PPTs treated showed significant differences between 
groups ( $\mathrm{p} \leq 0.05)$ (Figure $3 \mathrm{~B}-\mathrm{E}$ and Supplementary Figure S2C, and one aditional (Supraspinitus right) showed a tendency $(\mathrm{p}<0.1)$ (Supplementary Figure S2B), indicating differential response to treatment between groups. By contrast, none of the untreated PPTs showed any subgroup-associated difference ( $p>0.1$ ), with the exception of the Second rib right, which showed a tendency $(p<0.01)$ for differences to response between groups (Supplementary Figure S2F).
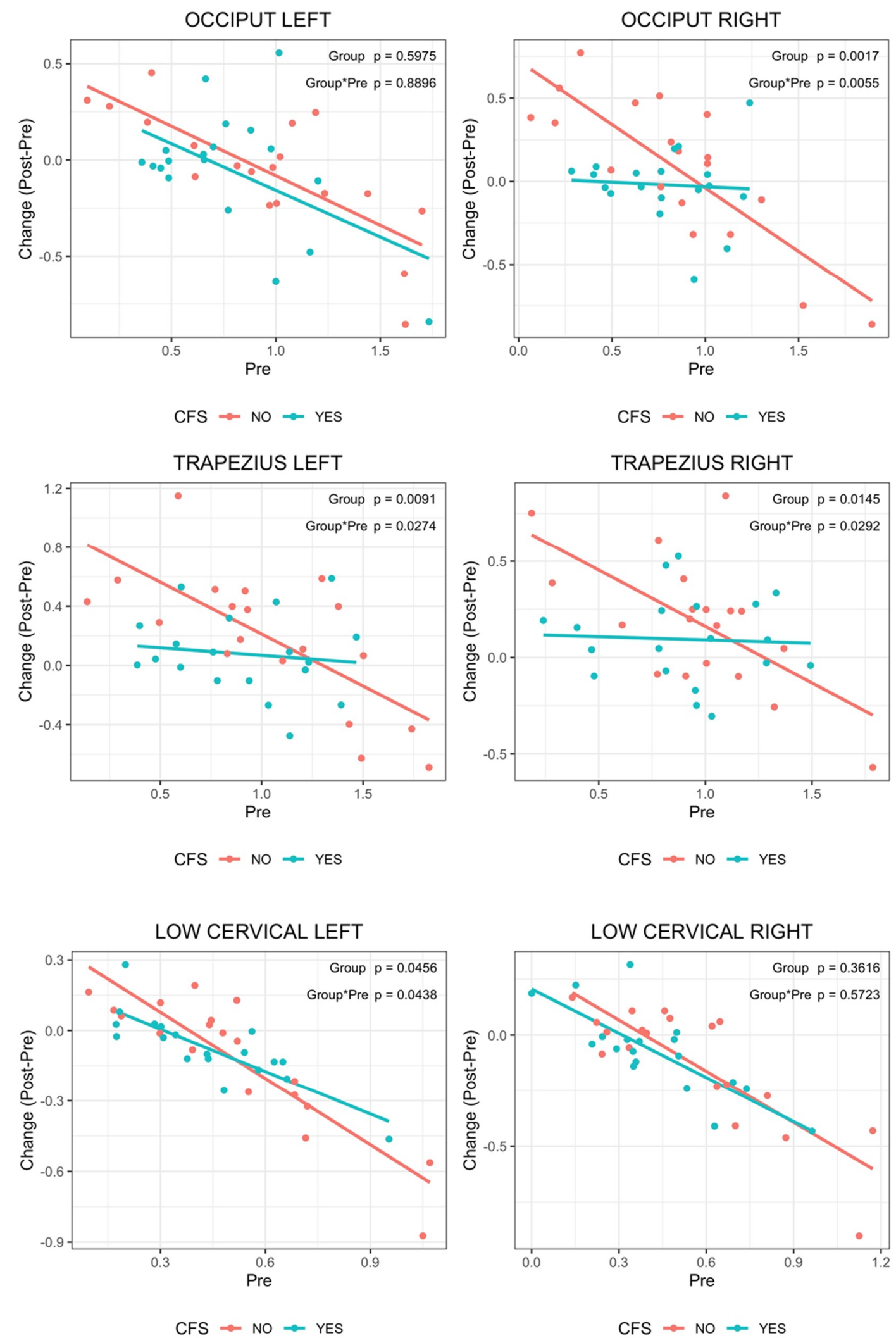

$\mathrm{CFS} \approx \mathrm{NO} \approx \mathrm{YES}$

$\mathrm{CFS} \approx \mathrm{NO} \approx \mathrm{YES}$ 
Figure 3. Linear dependence of PPT baseline scores (Pre) with MT therapy response (Change: Post-Pre) in patients presenting FM with or without comorbid ME/CFS. The plots show the inverse correlation between pre-treatment PPT values and symptom improvement, measured as the acquired resistance to pressure-induced pain for the tenderpoints at the Occiput left side of the body (A), or the right (B); and the same type of correlation for the tenderpoint at the left Trapezius (C) or its corresponding right PPT (D), and for the left Low cervical (E), or Low cervical right (F) . Linear adjustments in the FM only subgroup are shown in red color, while those for the FM patients that present with ME/CFS comorbidity are shown in green. P values of the differences between FM only and FM with ME/CFS are shown. 


\section{Discussion}

The possibility of drug-free therapeutics seems highly attractive, particularly for diseases like FM and ME/CFS that appear often associated to varied comorbidities, multiple chemical hypersensitivity included [47]. Also, for the expected low undesired secondary effects and the avoidance of harmful interactions.

The rationale behind the proposal of using pressure-controlled MT to treat FM and ME/CFS is extensively presented and carefully discussed in a review paper published by our group about 2 years ago [30]. Some of the data supporting this proposal includes several systematic reviews and meta-analysis of RCTs concluding that MT leads to beneficial effects on improving pain, anxiety and depression [28,30,48]. Another meta-analysis, including 140 studies has shown that MT reduces DOMS (delayed onset muscle soreness) and fatigue after exercise, more effectively than compression garment, electrostimulation, stretching, immersion or cryotherapy, as evidenced by decrease in the muscle damage marker creatine kinase (CK) and in the inflammation markers interleukin-6 (IL-6) and C-reactive protein [49], and therefore further supports MT as a potential therapy for FM.

MT activates mechano-transduction signaling pathways, induces mitochondria biogenesis signaling and diminishes the levels of inflammatory cytokines, such as the interleukin IL-6 and TNF(tumor necrosis factor)- $\alpha$ [50], changes that could benefit FM and CFS/ME patients [51,52]. Application of MT to soft and connective tissues leads to biochemical changes, at the time that allows local nociceptive and inflammatory mediators be reabsorbed by raising muscle blood and lymph circulation [53]. In fact, the use of pressure mimetic devices on animal models have shown that the intensity of therapeutic forces is determinant to obtain hypoalgesic effects [31,54]. Waters-Banker et al., by assaying low $(1.4 \mathrm{~N})$, medium $(4.5 \mathrm{~N})$, and high $(11 \mathrm{~N})$ loadings, found that high loadings drive to increased extracellular space possibly as result of damage and edema of the muscle. While medium loading induced anti-inflammatory molecular changes and induced muscle regeneration, low pressures did not [31], indicative of the pertinence of a moderate controlled pressure MT protocol as the choice with therapeutic potential.

In compliance with these findings, some MT studies have strived to control the applied load, towards effect replicability, using approaches like patient pain feed-back scales, as for example includes the recently published work by Nadal-Nicolás et al. [55]. Since pain sensitivity varies across FM patients, we thought that pressure monitorization performed by a single pre-trained collegiate physiotherapist might increase inter-assay consistency. For this purpose, we developed a pressuresensitive dispositive with built-in sensors that was used to ensure medium load MT protocols by selftraining (please see Methods for further details).

In line with our expectations, our medium load-pressure MT protocol consisting on 8-sessions of 25 mins twice weekly for 4 consecutive days on alternate weekdays (Figure 1), led to improvement of patient pain thresholds, as measured by the standard FIQ and SF-36 questionnaires (Table 3). FIQ total and Overload were the most improved items, together with SF-36 Bodily pain. Similarly, CQ results showed most benefits for response immediately after treatment and upon completion of the whole set of 8 -sessions $(\mathrm{p}<0.05)$, in contrast to $24 \mathrm{~h}$ after treatment, when patients reported worse condition of the treated region (Table 4). It is possible that at that time (24h post-treatment) some pressure-induced events lead to this feeling. It may be of interest to monitor patient evolution after treatment more closely in future trials. By contrast, no improvement was registered on patient's fatigue, either by MFI or CQ scores (Tables 3 and 4). As per the stability of the benefits obtained in pain reduction and overall status, we found that the effects persisted after a period free of treatment of 30-day (Tables 3 and 4), at least to a certain extent. Longer patient follow ups are granted by continuation studies to determine MT effects washout period. 
In agreement with the described questionnaire-assessed findings, differential PPTs measures obtained with a digital algometer, as described in Methods, further confirmed significant improvement of patient's hyperalgesia/allodynia in two of the treated sets of tender points, the Trapezius and the Gluteal. Only the Low cervical appear to worsen in the overall group assessment (Table 2). None of the untreated point PPTs were majorly affected with the only exception of the right Second rib, when the whole group was considered $(\mathrm{N}=38)$, suggesting that the improvement was as an overall limited to the areas receiving the MT treatment. This disagrees with the compressing benefits reported by some authors, who evidenced systemic improvement on contralateral untreated limbs in experimental animal models [31,32]. However, when improvement PPT scores (difference between Post and Pre- PPT values: POST-PRE on Figure 2 and Supplementary Figure S1), were plotted, we observed patients that were responders (POST-PRE values $>0$ ) and others that appeared as not responders (POST-PRE values $<0$ ), involving all, treated and untreated tender points (Figure 2 and Supplementary Figure S1), indicating two different type of patients, and also evidencing the existence of systemic effects.

In reference to the appreciated effects on untreated areas, and its interpretation, we must point that a limitation of this study is the impossibility to attribute the applied mechanical loads to the effects obtained due to the absence of a sham treated arm (group of patients treated with a similar MT protocol using a low $<1.4 \mathrm{~N}$ pressures) in our design (Figure 1 ). This arm was not included in this initial trial since our primary interest was to test the overall benefits and the performance of the output variables before investing any additional efforts. MT treatments inherently contain an emotional component, transferred to the patient through mental relaxation and stress reduction by the sense of touch. In line with this, several are the authors that have demonstrated beneficial health effects obtained from positive emotional stimulus, either reflected by the boosting of the immune system [35, 56,57], and effects in weight gain and increased vagal tone [58], among other. Future interventions should add a sham group to discern mechanical from emotional triggered effects.

Unexpectedly, a negative correlation between patient's initial hyperalgesia/allodynia status and MT therapeutic benefit was evidenced when the Change Post-Pre values were plotted as a function of their PPT baseline (Pre) values (Figure 2 and Supplementary Figure S1). This importantly points out that patient sensitivity to pain, as assessed by its PPTs with an algometer, could serve as an indicator or predictor of the expected patient's response to MT therapy. In particular, the data indicates that FM patients with worse health status (or at least with highest sensitivity to pain) will obtain most benefits while those presenting closer to normal pain threshold levels will not experiment improvements or will improve to a lesser extent. Among all 18 tender points assayed, we found that the Low cervical, in the treated group, and the Knee within the untreated, constitute the best sensors for this prediction, with prediction values of around $60 \%$, reaching $74 \%$ individually (Figure 2 and Supplementary Figure S1).

Moreover, we observed that while patients presenting only FM followed PPT improvement patterns inversely correlated with their baseline hyperalgesia/allodynia status, those diagnosed with comorbid ME/CFS did not respond to MT, at least for some of the treated points, as indicated by an overall lack of change in PPT values (Figure 3 B-E, green lines). This suggests different responses for patients diagnosed with FM only with respect to those presenting comorbid ME/CFS, which is further supported by the obtained CQ scores (Tables 6 and 7).

These observations open the unprecedented possibility of classifying patients as potential "responders" or "not responders" to pressure-controlled MT, based on their thresholds to pain and the presence of comorbid ME/CFS, further supporting somatic differences and mechanisms behind pain perception in one or the other disease. Nevertheless, since the number of observations here is limited, and the current trial the first to detect these correlations, the findings will need to be validated in additional, more numerous cohorts. 
In reference to the studied cohort it should be stressed that the participating patients were mainly females (35/38), with an average age of $55.6 \pm 7.2$ years (range 43-71) and time from primary FM diagnosis over 3 years. Most (79\%) presented severe affection while $21 \%$ corresponded to moderate cases. Physical problems overweighed emotional (Table 1). In addition, half of them had received diagnosis of ME/CFS. Potential immune and metabolic unbalance associated with low neutrophil percentages ( $63.8 \%$ of the participants), increased monocytes $(41.6 \%)$, high lymphocytes $(16.7 \%)$, high cholesterol $(72.2 \%)$ and low LDL $(40 \%)$, may be present. Increased levels of creatinine could relate to muscle damage in $22.9 \%$ of the participants (Supplementary Table S2, N=36). The possibility that different cohorts of patients lead to distinct outcomes after applying this same protocol remains.

Differences between the participants having been diagnosed with FM only and those receiving diagnosis of both FM and ME/CFS at baseline were identified, mainly with the FIQ items Overall and Symptoms, and with the SF-36 Physical and Vitality items, MFI failed in detecting differences between these two groups. Similarly, FIQ and SF-36 were also superior at detecting differences between the response to MT of either group, with Overall and Total for the first questionnaire and Vitality, Social Functioning and Mental Health in the second. The differences found between groups 30-day after treatment withdrawal could relate to the mentioned observations of distinct response and the fact that the 30-day withdrawal seem not to be enough to washout treatment effects, mostly affecting again to physical roles, as assessed by the SF-36 instrument (Table 5). Thus, FIQ scores and SF-36 Vitality values may turn into additional indicators of the presence of ME/CFS comorbidity in FM.

Finally, the value of the collection of blood samples gathered and Biobanked in the context of this study results evident, as particular molecular profiles from some of the sets should permit revealing the cellular pathways mediating the response to this pressure controlled-MT protocol, as previously envisioned by our group [30]. Importantly differential molecular profiles will also allow us to understand the reasons behind patient's response dependence according to patient's hypoalgesia/allodynia status and with the co-diagnosis of ME/CFS. It is quite likely that these future studies yield new biomarkers for the prediction of response to MT, some perhaps allowing differential diagnosis of the FM and the ME/CFS disease.

\section{Conclusions}

The results from this single arm interventional CT based on a 8-session pressure-controlled manual therapy allow us to conclude that FM patients response to MT treatment is dependent on the initial hyperalgesia/allodynia status of the patient, and on ME/CFS comorbidity, with improved outcomes for patients with increased pain sensitivity, as determined by PPT measurements; and for those presenting FM in the absence of concomitant ME/CFS.

Therefore, it is proposed that baseline PPT values and the diagnosis of concurrent ME/CFS could serve as criteria to predict patient response to pressure-controlled physiotherapy programs. In addition, standard FIQ and SF-36 itemized scores, individually or in conjunction with our CQ questionnaire might help detect ME/CFS within FM cohorts, and perhaps serve to identify responders from those that do not respond to this type of mechanically triggered therapy.

Since a complete collection of blood samples is available from this study, it is expected that future molecular evaluations reveal the subjacent mechanisms to the identified patient response to MT treatment among other.

Supplementary Materials: The following are available online at www.mdpi.com/xxx/s1, Figure S1: title, Table S1: title, Video S1: title.

Author Contributions: Conceptualization and methodology M.G-E. and E.O.; investigation, F.J.F-V. and J.B-F..; data curation and analysis F.J.F-V; M.Z. and E.O.; writing-original draft preparation, F.J.F-V. and E.O.; funding 
acquisition and supervision, M.G-E and E.O. All authors have read, reviewed and edit and agreed to the published version of the manuscript.

Funding: This research was funded by the CITSAM (Centro de Investigación San Alberto Magno), Universidad Católica de Valencia San Vicente Mártir, grant numbers 2019-266-001 and 2019-270-002.

Acknowledgments: We are grateful to the collegiate nurse Ignacio Bonastre Ferez for his help with the phlebotomies, to Teresa Sánchez-Fito for technical support in the laboratory, to the Universidad Católica de Valencia Clinics personnel for their help with patient registration and appointment follow-ups, and to all volunteers that participated in the study.

Conflicts of Interest: The authors declare no conflict of interest. The funders had no role in the design of the study; in the collection, analyses, or interpretation of data; in the writing of the manuscript, or in the decision to publish the results. 


\section{References}

1. Boerma, T.; Harrison, J.; Jakob, R.; Mathers, C.; Schmider, A.; Weber, S. Revising the ICD: Explaining the WHO approach. Lancet 2016, 388, 2476-2477.

2. Wolfe, F.; Smythe, H.A.; Yunus, M.B.; Bennett, R.M.; Bombardier, C.; Goldenberg, D.L. The American College of Rheumatology 1990 Criteria for the Classification of fibromyalgia. Report of the Multicenter Criteria Committee. Arthritis Rheum 1990, 33, 160-172.

3. Wolfe, F.; Clauw, D.J.; Fitzcharles, M.A. The American College of Rheumatology preliminary diagnostic criteria for fibromyalgia and measurement of symptom severity. Arthritis Care Res. 2010, 62, 600-610.

4. Jahan, F.; Nanji, K.; Qidwai, W.; Qasim, R. Fibromyalgia syndrome: An overview of pathophysiology, diagnosis and management. Oman Med. J. 2012, 27, 192-195.

5. Carruthers, B.M.; Jain, A.K.; De Meirleir, K.L.; Peterson, D.L.; Klimas, N.G.; Lerner, A. Myalgic Encephalomyelitis/Chronic Fatigue Syndrome: Clinical Working Case Definition, Diagnostic and Treatment Protocols. J. Chronic Fatigue Syndrome 2003, 11, 7-115.

6. Carruthers, B.M.; van de Sande, M.I.; De Meirleir, K.L.; Klimas, N.G.; Broderick, G.; Mitchell, T. Myalgic encephalomyelitis: International Consensus Criteria. J. Intern. Med. 2011, 270, 327-338.

7. Barsky, A.J.; Borus, J.F. Functional somatic syndromes. Ann. Intern. Med. 1999, 130, 910921.

8. Abbi, B.; Natelson, B.H. Is chronic fatigue syndrome the same illness as fibromyalgia: Evaluating the 'single syndrome' hypothesis. QJM. 2013, 106, 3-9.

9. Natelson BH. Myalgic Encephalomyelitis/Chronic Fatigue Syndrome and Fibromyalgia: Definitions, Similarities, and Differences. Clin Ther. 2019;41(4):612-618. doi:10.1016/j.clinthera.2018.12.016

10. Natelson, B.H.; Vu, D.; Coplan, J.D.; Mao, X.; Blate, M.; Kang, G.; Soto, E.; Kapusuz, T.; Shungu, D.C. Elevations of Ventricular Lactate Levels Occur in Both Chronic Fatigue Syndrome and Fibromyalgia. Fatigue 2017, 5, 15-20.

11. Ciccone, D.S.; Natelson, B.H. Comorbid illness in women with chronic fatigue syndrome: a test of the single syndrome hypothesis. Psychosom Med. 2003, 65(2), 268-275.

12. McManimen, S.L.; Jason, L.A. Post-Exertional Malaise in Patients with ME and CFS with Comorbid Fibromyalgia. SRL Neurol. Neurosurg. 2017, 3, 22-27.

13. Naschitz, J.E.; Rozenbaum, M.; Rosner, I.; Sabo, E.; Priselac, R.M.; Shaviv, N. Cardiovascular response to upright tilt in fibromyalgia differs from that in chronic fatigue syndrome. J. Rheumatol. 2001, 28, 1356-1360.

14. Naschitz, J.E.; Slobodin, G.; Sharif, D.; Fields, M.; Isseroff, H.; Sabo, E. Electrocardiographic QT interval and cardiovascular reactivity in fibromy yalgia differ from chronic fatigue syndrome. Eur. J. Intern Med. 2008, 19,187-191. 
15. Korszun, A.; Sackett-Lundeen, L.; Papadopoulos, E.; Brucksch, C.; Masterson, L.; Engelberg, N.C. Melatonin levels in women with fibromyalgia and chronic fatigue syndrome. J. Rheumatol. 1999, 26, 2675-2680.

16. Crofford, L.J.; Young, E.A.; Engleberg, N.C.; Korszun, A.; Brucksch, C.B.; McClure, L.A. Basal circadian and pulsatile ACTH and cortisol secretion in patients with fibromyalgia and/or chronic fatigue syndrome. Brain Behav. Immun. 2004, 18, 314-325.

17. Light, A.R.; Bateman, L.; Jo, D.; Hughen, R.W.; Vanhaitsma, T.A.; White, A.T.; Light, K.C. Gene expression alterations at baseline and following moderate exercise in patients with Chronic Fatigue Syndrome and Fibromyalgia Syndrome. J. Intern. Med. 2012, 271, 64-81.

18. Cerda-Olmedo, G.; Mena-Duran, A.V.; Monsalve, V.; Oltra, E. Identification of a microRNA signature for the diagnosis of fibromyalgia. PLoS ONE 2015, 10, e0121903.

19. Masotti, A.; Baldassarre, A.; Guzzo, M.P.; Iannuccelli, C.; Barbato, C.; Di Franco, M. Circulating microRNA Profiles as Liquid Biopsies for the Characterization and Diagnosis of Fibromyalgia Syndrome. Mol. Neurobiol. 2017, 54, 7129-7136.

20. Kwiatek, R. Treatment of fibromyalgia. Aust. Prescr. 2017, 40, 179-183.

21. Bruun Wyller, V.; Bjørneklett, A.; Brubakk, O.; Festvåg, L.; Follestad, I.; Malt, U. Diagnosis and Treatment of Chronic Fatigue Syndrome/Myalgic Encephalopathy (CFS/ME) [Internet]. Oslo, Norway: Knowledge Centre for the Health Services at The Norwegian Institute of Public Health (NIPH); 2006. Available online: http://www.ncbi.nlm.nih.gov/books/NBK464796/.

22. Smith, M.E.; Haney, E.; McDonagh, M.; Pappas, M.; Daeges, M.; Wasson, N.; Fu, R.; Nelson, H.D. Treatment of Myalgic Encephalomyelitis/Chronic Fatigue Syndrome: A Systematic Review for a National Institutes of Health Pathways to Prevention Workshop. Ann. Intern. Med. 2015, 162, 841-850.

23. Busch, A.J.; Webber, S.C.; Brachaniec, M.; Bidonde, J.; Bello-Haas, V.D.; Danyliw, A.D. Exercise therapy for fibromyalgia. Curr. Pain Headache Rep. 2011, 15, 358-367.

24. Twisk, F. PACE: CBT and GET are not rehabilitative therapies. Lancet Psychiatry 2016, 3, e6.

25. Wilshire, C.E.; Kindlon, T.; Courtney, R.; Matthees, A.; Tuller, D.; Geraghty, K. Rethinking the treatment of chronic fatigue syndrome-a reanalysis and evaluation of findings from a recent major trial of graded exercise and CBT. BMC Psychol. 2018, 6, 6.

26. Field, T.; Diego, M.; Hernandez-Reif, M. Moderate pressure is essential for massage therapy effects. Int. J. Neurosci. 2010, 120, 381-385.

27. Field, T.; Hernandez-Reif, M.; Diego, M.; Schanberg, S.; Kuhn, C. Cortisol decreases and serotonin and dopamine increase following massage therapy. Int. J. Neurosci. 2005, 115, 1397-1413.

28. Li, Y.H.; Wang, F.Y.; Feng, C.Q.; Yang, X.F.; Sun, Y.H. Massage therapy for fibromyalgia: A systematic review and meta-analysis of randomized controlled trials. PLoS ONE 2014, 9, e89304. 
29. Yuan, S.L.; Matsutani, L.A.; Marques, A.P. Effectiveness of different styles of massage therapy in fibromyalgia: A systematic review and meta-analysis. Man Ther. 2015, 20, 257264.

30. Espejo, J.A.; García-Escudero, M.; Oltra, E. Unraveling the Molecular Determinants of Manual Therapy: An Approach to Integrative Therapeutics for the Treatment of Fibromyalgia and Chronic Fatigue Syndrome/Myalgic Encephalomyelitis. Int J Mol Sci. 2018, 19(9), 2673.

31. Waters-Banker, C.; Butterfield, T.A.; Dupont-Versteegden, E.E. Immunomodulatory effects of massage on nonperturbed skeletal muscle in rats. J Appl Physiol. 2014, 116(2), 164-75.

32. Miller, B.F.; Hamilton, K.L.; Majeed, Z.R.; Abshire, S.M.; Confides, A.L.; Hayek, A.M. Enhanced skeletal muscle regrowth and remodelling in massaged and contralateral nonmassaged hindlimb. J. Physiol. 2018, 596, 83-103.

33. Sagar, S.M.; Dryden, T.; Wong, R.K. Massage therapy for cancer patients: A reciprocal relationship between body and mind. Curr. Oncol. 2007, 14, 45-56.

34. Fang, C.Y.; Reibel, D.K.; Longacre, M.L.; Rosenzweig, S.; Campbell, D.E.; Douglas, S.D. Enhanced psychosocial well-being following participation in a mindfulness-based stress reduction program is associated with increased natural killer cell activity. J. Altern. Complement. Med. 2010, 16, 531-538.

35. D'Acquisto, F. Affective immunology: where emotions and the immune response converge. Dialogues Clin Neurosci. 2017, 19(1), 9-19.

36. Kumar, A.; Chaudhry, I.; Reid, M.B.; Boriek, A.M. Distinct signaling pathways are activated in response to mechanical stress applied axially and transversely to skeletal muscle fibers. J. Biol. Chem. 2002, 277, 46493-46503.

37. Kumar, A.; Boriek, A.M. Mechanical stress activates the nuclear factor-kappaB pathway in skeletal muscle fibers: A possible role in Duchenne muscular dystrophy. FASEB J. 2003, 17, 386-396.

38. Burckhardt, C.S.; Clark, S.R.; Bennett, R.M. The fibromyalgia impact questionnaire: Development and validation. J. Rheumatol. 1991, 18, 728-733.

39. Rivera, J.; González, T. The Fibromyalgia Impact Questionnaire: A validated Spanish version to assess the health status in women with fibromyalgia. Clin. Exp. Rheumatol. 2004, $22,554-560$.

40. Smets, E.; Garssen, B.; Bonke, B.; De Haes, J. The multidimensional Fatigue Inventory (MFI) psychometric qualities of an instrument to assess fatigue. J. Psychosom. Res. 1995, 39, 315325.

41. McHorney, C.A.; Ware, J.E., Jr.; Raczek, A.E. The MOS 36-Item Short-Form Health Survey (SF-36): II. Psychometric and clinical tests of validity in measuring physical and mental health constructs. Med. Care 1993, 31, 247-263.

42. Jones, D.H.; Kilgour, R.D.; Comtois, A.S. Test-Retest Reliability of Pressure Pain Threshold Measurements of the Upper Limb and Torso in Young Healthy Women. J Pain. 2007, 8(8), 650-6. 
43. Fryer, G.; Hodgson, L. The effect of manual pressure release on myofascial trigger points in the upper trapezius muscle. J Bodyw Mov Ther. 2005, 9(4), 248-55.

44. Ovejero, T.; Sadones, O.; Sánchez-Fito, T., et al. Activation of Transposable Elements in Immune Cells of Fibromyalgia Patients. Int J Mol Sci. 2020, 21(4), 1366.

45. R Core Team (2019). R: A language and environment for statistical computing. $R$ Foundation for Statistical Computing, Vienna, Austria. URL https://www.R-project.org/

46. H. Wickham. ggplot2: Elegant Graphics for Data Analysis. Springer-Verlag New York, 2016

47. Castro-Marrero, J.; Faro, M.; Aliste, L., et al. Comorbidity in Chronic Fatigue Syndrome/Myalgic Encephalomyelitis: A Nationwide Population-Based Cohort Study. Psychosomatics. 2017, 58(5), 533-543.

48. Crawford, C.; Boyd, C.; Paat, C.F.; Price, A.; Xenakis, L.; Yang, E. Evidence for Massage Therapy (EMT) Working Group. The Impact of Massage Therapy on Function in Pain Populations-A Systematic Review and Meta-Analysis of Randomized Controlled Trials: Part, I., Patients Experiencing Pain in the General Population. Pain Med. 2016, 17, 13531375.

49. Dupuy, O.; Douzi, W.; Theurot, D.; Bosquet, L.; Dugué, B. An Evidence-Based Approach for Choosing Post-exercise Recovery Techniques to Reduce Markers of Muscle Damage, Soreness, Fatigue, and Inflammation: A Systematic Review with Meta-Analysis. Front. Physiol. 2018, 9, 403.

50. Crane, J.D.; Ogborn, D.I.; Cupido, C.; Melov, S.; Hubbard, A.; Bourgeois, J.M. Massage therapy attenuates inflammatory signaling after exercise-induced muscle damage. Sci. Transl. Med. 2012, 4, 113-119.

51. Tsilioni, I.; Russell, I.J.; Stewart, J.M.; Gleason, R.M.; Theoharides, T.C. Neuropeptides CRH, SP, HK-1, and Inflammatory Cytokines IL-6 and TNF Are Increased in Serum of Patients with Fibromyalgia Syndrome, Implicating Mast Cells. J. Pharmacol. Exp. Ther. 2016, 356, 664-672.

52. Patarca, R.; Klimas, N.G.; Lugtendorf, S.; Antoni, M.; Fletcher, M.A. Dysregulated expression of tumor necrosis factor in chronic fatigue syndrome: Interrelations with cellular sources and patterns of soluble immune mediator expression. Clin. Infect. Dis. 1994, 18, S43-S53.

53. Goats, G.C. Massage-the scientific basis of an ancient art: Part 2. Physiological and therapeutic effects. Br. J. Sports Med. 1994, 28, 153-156.

54. McLean, S.; Naish, R.; Reed, L.; Urry, S.; Vicenzino, B. A pilot study of the manual force levels required to produce manipulation induced hypoalgesia. Clin. Biomech. 2002, 17, 304308.

55. Nadal-Nicolás, Y.; Rubio-Arias, J.Á.; Martínez-Olcina, M.; Reche-García, C.; HernándezGarcía, M.; Martínez-Rodríguez, A. Effects of Manual Therapy on Fatigue, Pain, and Psychological Aspects in Women with Fibromyalgia. Int J Environ Res Public Health. 2020, 17(12), 4611. 
56. Berk, L.S.; Felten, D.L.; Tan, S.A.; Bittman, B.B.; Westengard, J. Modulation of neuroimmune parameters during the eustress of humor-associated mirthful laughter. Altern. Ther. Health Med. 2001, 7, 62-72.

57. Fang, C.Y.; Reibel, D.K.; Longacre, M.L.; Rosenzweig, S.; Campbell, D.E.; Douglas, S.D. Enhanced psychosocial well-being following participation in a mindfulness-based stress reduction program is associated with increased natural killer cell activity. J. Altern. Complement. Med. 2010, 16, 531-538.

58. Niemi, A.K. Review of Randomized Controlled Trials of Massage in Preterm Infants. Children 2017, 4 